\title{
Research on 3D Rendering Effect of Marine Bionic Packaging Container Based on Deep Learning and Visualization
}

\author{
Jie Ning $(\mathbb{D}$, Xiaonan Ren, and Joung Hyung Cho $\mathbb{i}$ \\ Department of Marine Design Convergence Engineering, Pukyong National University, Busan 48513, Republic of Korea \\ Correspondence should be addressed to Joung Hyung Cho; jhcho7@pknu.ac.kr
}

Received 5 November 2021; Revised 13 December 2021; Accepted 22 December 2021; Published 17 February 2022

Academic Editor: Man Fai Leung

Copyright ( $) 2022$ Jie Ning et al. This is an open access article distributed under the Creative Commons Attribution License, which permits unrestricted use, distribution, and reproduction in any medium, provided the original work is properly cited.

\begin{abstract}
Three-dimensional rendering includes the test of system development environment and running environment. Three-dimensional rendering influencing factors test: the number and size of parallel blocks affect the average rendering frame rate of 26.9 when the number is 9600 and the size is $10 \times 10$, and the average rendering frame rate is 13.7 when the number is 600 and the size is $40 \times 40$. According to relevant data, it is inferred that the average rendering frame rate is higher when the number of parallel blocks is large and the size of parallel blocks is small, and the number is inversely proportional to the size. The rendering window size also has a relative influence on the rendering frame rate. When the window size is $600 \times 400$, only the terrain frame rate is 77.3 , and when the window size is $1200 \times 800$, the frame rate is 68.1 , which shows that when only the terrain frame rate is rendered, the frame rate decreases with the increase in the window size, and its performance is not high when only CPU is used. However, when CPU is combined with GPU, the performance is greatly improved, reaching the highest of 29.3 and the lowest of 21.5 , which is much higher than that when only CPU is used. According to the influence of the number of sampling points on the frame rate, the $5 \times 5$ sampling method and $2 \times 2$ sampling method proposed in this paper have better frame rate performance than the traditional template shadow method. Through the above data, we know that the number of parallel blocks, size, window size, sampling method, and other factors have a certain impact on the average rendering frame rate. Marine bionics is a marginal subject between marine biology and technical engineering science. Deep networks have had a huge impact on the field of machine learning research and application, but at the same time, they cannot clearly explain the ins and outs of deep networks. People have been working to understand the complex process more thoroughly. Since humans' cognition and experience of the world mainly come from vision, good visualization can effectively help people understand the deep network and perform effective optimization and adjustment.
\end{abstract}

\section{Introduction}

Through the analysis of deep learning and 3D rendering of marine bionic packaging containers under visualization, we know the deep neural network process of deep learning, visualization method, 3D rendering principle and process analysis, and marine bionic principle. By studying the deep learning function mode to improve visualization, 3D rendering principle, and process analysis accuracy, according to the factors affecting 3D rendering to improve its rendering performance, the marine bionic packaging container is manufactured through the principle of marine bionic so as to analyze its $3 \mathrm{D}$ rendering effect.
In literature [1], the topic of computer vision has recently had an impact on food detection and medical applications. The deep learning paradigm of computer vision object segmentation and the visual paradigm learning of online or offline intensive training are the main contents of this paper. Literature [2] reduced the growing number of malicious software by combining RNN with CNN. Learning from raw data [3], that is, deep learning, changes computer vision through end-to-end learning. Through research, we know that this method improves the decoding performance of deep convolution network, and the average decoding is improved from $82.1 \%$ to $84 \%$. Good performance is achieved. Literature [4] explored the ability of some microorganisms to manipulate host behavior through the serial 
block scanning electron microscope and visual image segmentation algorithm based on deep learning. Literature [5] solved the problem of the visualization method of driving behavior by measuring driving behavior with various types of sensors. In order to help people identify the unique driving patterns in continuous driving, we set up driving behavior visualization. In order to distinguish malware and reduce the threat of malware to the Internet [6], we use the MCSC static feature method to transform malware code into gray image for recognition. Experiments show that the MCSC method is very effective in reducing Internet malware, and it can also be combined with main block selection to improve resolution. Literature [7] was proposed to promote the absorption of trace metals, iron in stomach and manganese and zinc in intestine in seaweed decoction. Biomimetic digestion in vitro and biomimetic biofilm extraction can be realized by biomimetic technology, and the absorption of organic matter in stomach and intestine can be promoted. Literature [8] showed that the potential information revealed by VEA learning crystal structure is obtained through VAE and XRD through simulation and experimental data analysis. At the same time, this method can effectively classify XRD data and can quickly identify data outside the distribution, such as new phase and mixture, all of which reflect the value of the VEA method. The YOLO deep learning model combined with the block matching algorithm locates myocardial wall [9], obtains its flow velocity, and draws eddy current graph by nonlinear weight function. The method of YOLO vectorogram analysis and evaluation is based on the visualization analysis of cardiac fluid movement and combined with the analysis of ultrasonic images of cardiac flow field. Finally, the correctness of the proposed method is verified by experiments, which provides a new basis Literature [10] was proposed to solve the problem of limited number of labeled medical images. The lung inflammation caused by COVID-19 was effectively distinguished from other lung inflammations by implementing transfer learning pipeline, and the overall detection accuracy was $90 \%, 94.3 \%$, and $96.8 \%$. Literature [11] learnt the mapping through the minimum primitive space method of deep neural network, which is a visual learning method. The visualization ability of neural network can be improved by training additional virtual data of neural network and using new activation function of neural network. In literature [12], iIn order to get the best visualization effect, the shadow method combination can be used. Four methods are used to detect the quality of the surface coloring algorithm: z-buffer gradient, gray gradient, adaptive gray gradient, and two extended cubes. In contrast, except for thin objects, gray gradient has stronger ability. Literature [13] proposed $3 \mathrm{D}$ rendering of $\mathrm{VR}, \mathrm{SSD}$, and maximum intensity projection of computed tomography data to scan for many years. Among them, MPR is useful for identifying small cracks, VR is useful for identifying bare ankle tendons, and 3D is helpful for patients with arthritis. In literature [14], hydrocephalus can be tested by $3 \mathrm{D}$ rendering, and surface rendering and conventional linear CSF imaging are valuable for judgment. Compared with planar scanning, it has the advantages of improving high-quality images and more intuitive and clear judgment of disease condition, but its diagnostic value estimation needs further investigation. Literature [15] showed that $3 \mathrm{D}$ rendering is especially suitable for free-view video applications. The new depth map compression strategy introduces an effective compression strategy for the shape of the segmented region and then predicts the surface shape from the segmented region and a group of samples with regular intervals, so as to effectively compress a few prediction residuals. The marine bionic packaging container is limited by the actual application scene, and the biological movement scene in the marine environment is realized by simulating the $3 \mathrm{D}$ technology. This article mainly highlights the practical application scenarios that this kind of $3 \mathrm{D}$ can achieve. In the literature research, there are many application scenarios of 3D technology, but the application scenarios considered are relatively single, and the application in more complex scenarios is not considered, especially the complex transformation of different application objects in ocean simulation scenarios. Therefore, it is of great significance to study the application of 3D technology in marine bionic packaging containers.

In this paper, 3D rendering effect, deep neural network, and visualization technology are combined to analyze the $3 \mathrm{D}$ rendering of deep learning and visualization of marine bionic packaging containers. By analyzing the influencing factors of $3 \mathrm{D}$ rendering (the size and number of parallel blocks, the size of rendering window, the amount of terrain data, and the number of sampling points), we use the LOD model to optimize $3 \mathrm{D}$ rendering, realize the relevant advantages of marine life simulation scene, and create a marine bionic packaging container suitable for human use.

\section{Research on 3D Rendering of Deep Learning and Visualization}

2.1. Deep Neural Network of Deep Learning. The input layer transmits the information to the hidden layer, and then the hidden layer transmits the data to the value output layer [16] as shown in Figures land 2.

2.2. Visualization Method and Process. The data set is processed, tested, trained, and verified [17], and the specific process is shown in Figures 3and 4.

By inputting data, the data are mapped to visualization and transformed into knowledge through user interaction in visualization [17]. Firstly, the data are mined to the model; secondly, the model is transformed into knowledge through parameter improvement; finally, the knowledge can also be recycled through feedback loop to form data again.

2.3. Three-Dimensional Rendering Analysis. The 3D application is passed to the $3 \mathrm{D}$ application program interface [18]. The GPU front-end program can transfer the loading of vertex data to the primitive assembly and change from point to point, line, and plane; finally, it is transferred to the cache, and the frame cache displays pixels at a time as shown in Figures 5 and 6. 


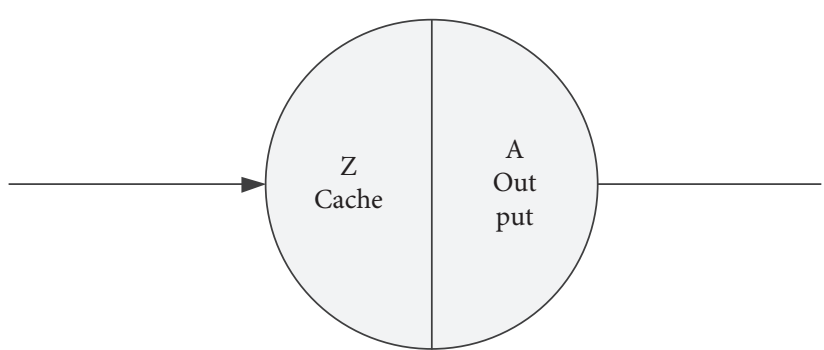

Figure 1: Node diagram of deep neural network for deep learning.

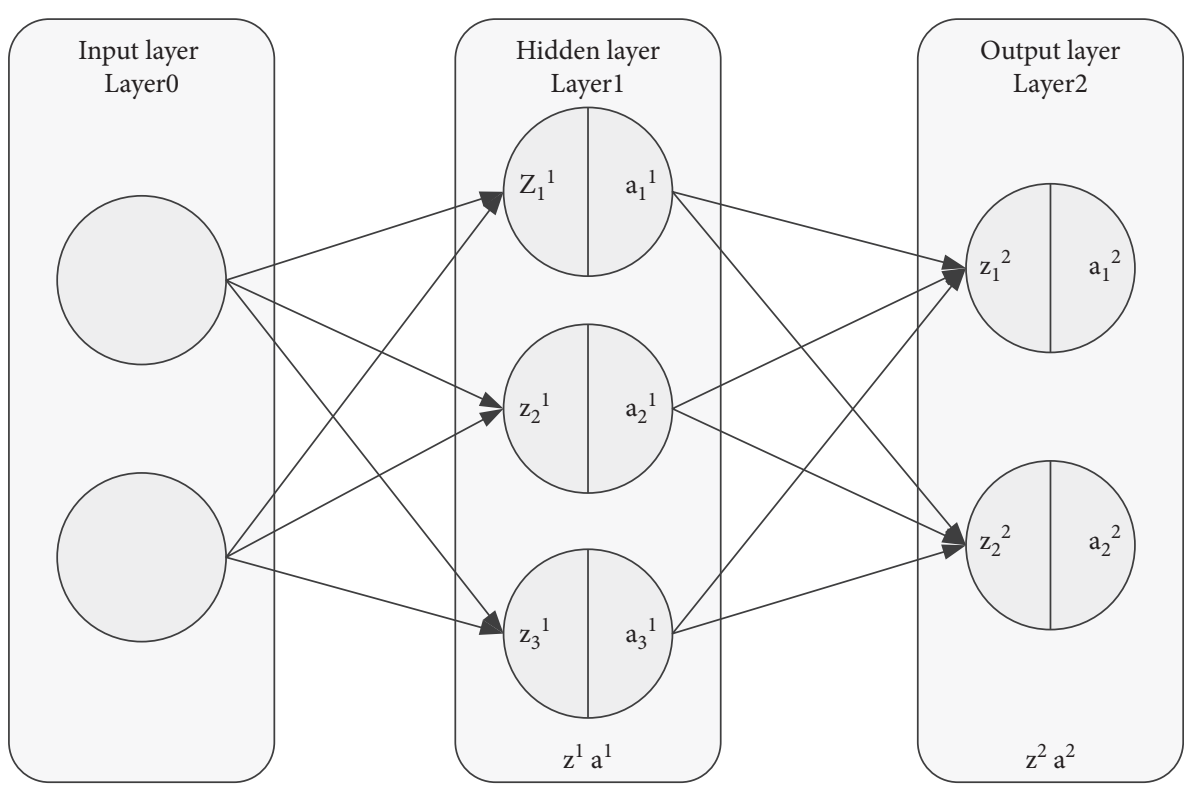

Figure 2: Basic structure diagram of deep neural network.

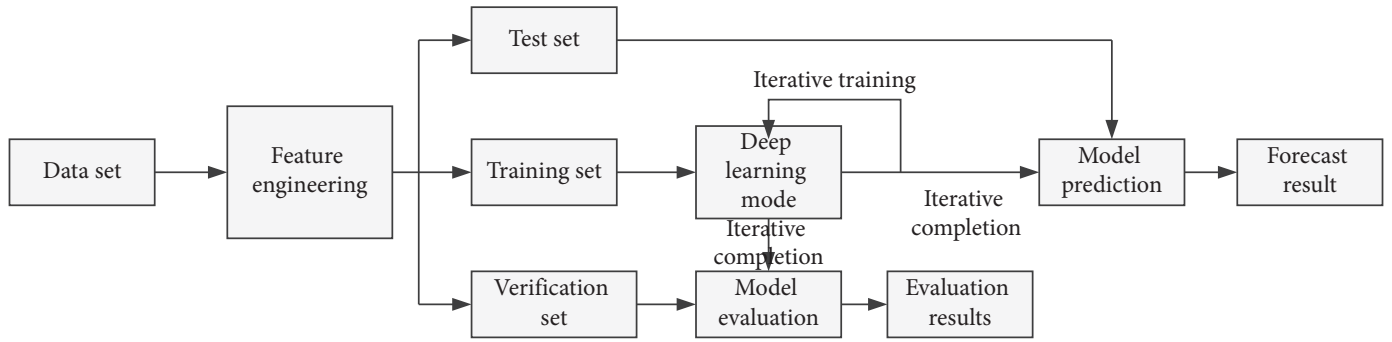

Figure 3: Flow chart of visual dataset.

Two-dimensional vector is transformed into the threedimensional coordinate system by the plane coordinate system and is transformed into the observation coordinate system by using the camera [19].

\subsection{Marine Bionics}

2.4.1. Principle of Marine Bionic Packaging Container. The problem of man and nature has always been a major concern of our human beings. In the long process of development, mankind has been constantly changing nature, and at the same time, it has been affected by nature. From the ancient primitive society to today, human beings have created their own colorful lifestyle and living environment through their own power, but these are all based on the use, change, and sacrifice of nature. In the long process of getting along with nature, many bionic creatures have been created through the influence of many creatures, and the average living standard of citizens has been continuously improved. Of course, these should be realized on the basis of peaceful coexistence between man and nature as shown in Figure 7.

Packaging container modeling design is a visual form that combines function with beauty and combines application with science to make packaging container a three-dimensional design. The processing technology made of paper, plastic, metallic glass, and other materials creates a three-dimensional form, so as to achieve the main purpose of holding, storing, protecting goods, and conveying information. 


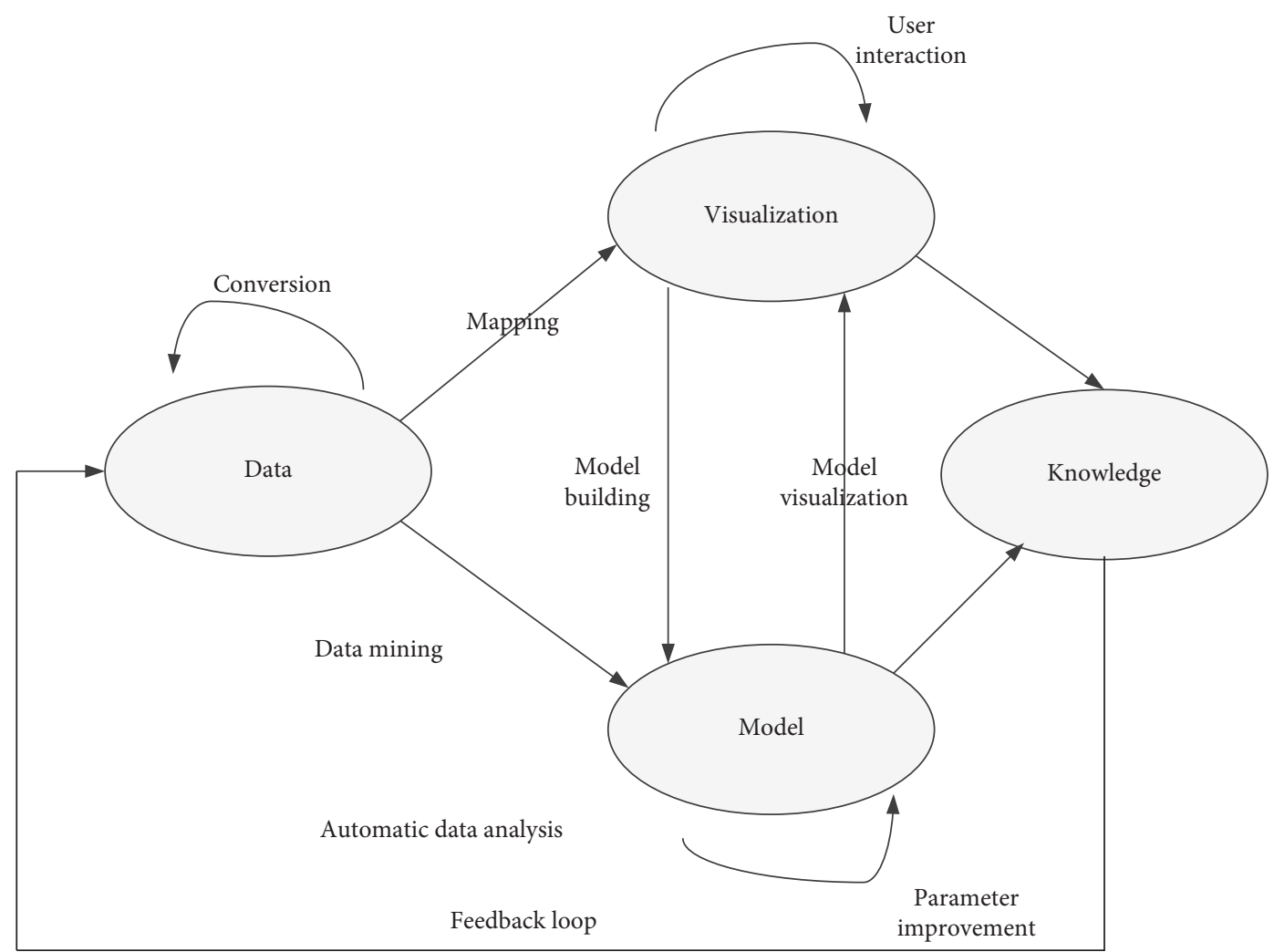

Figure 4: Visual data loop diagram.

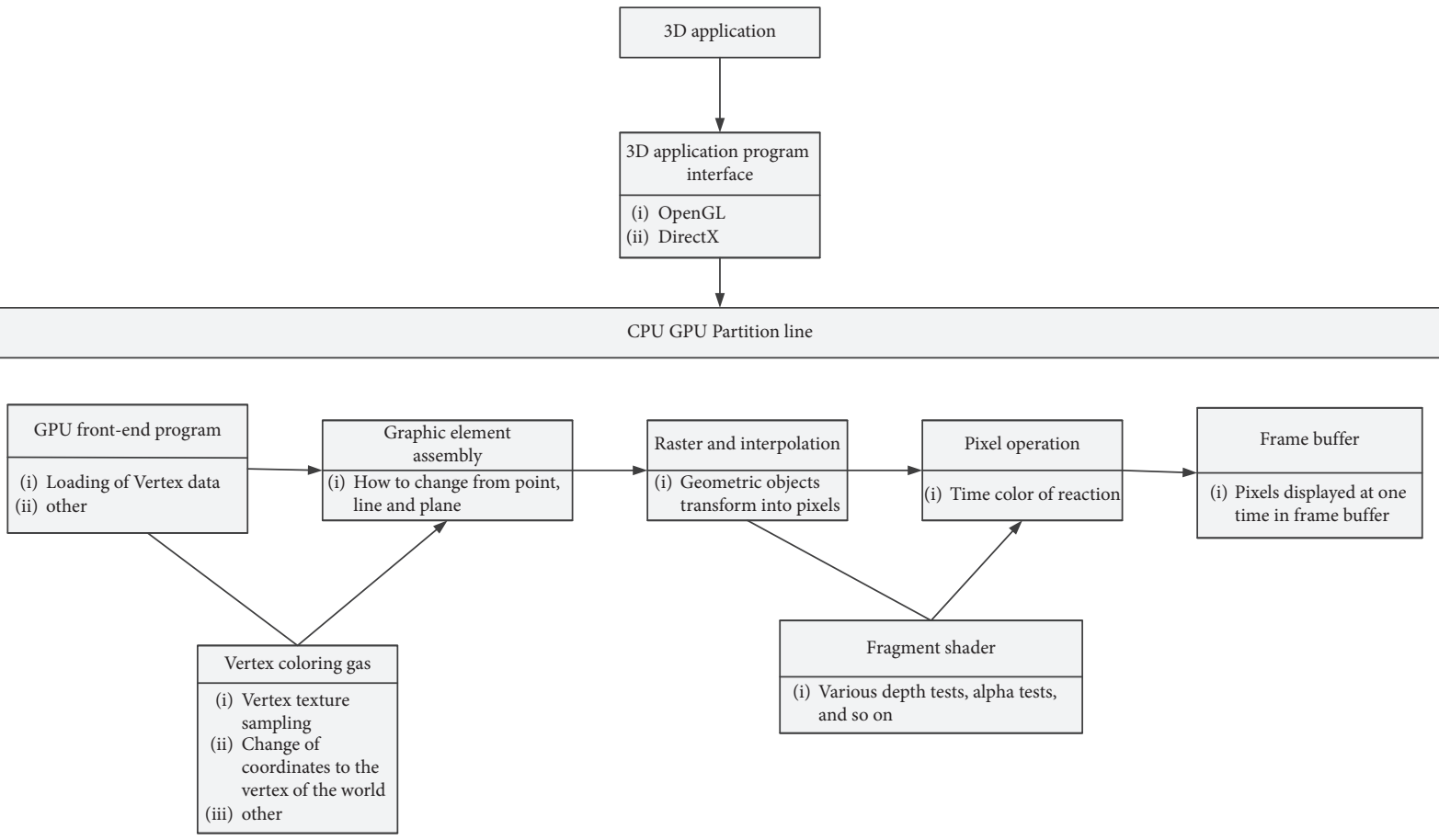

Figure 5: Flow chart of 3D rendering analysis.

2.4.2. Marine Bionic Process. First of all, we collect the experimental data of marine life movement, then scan it by using $3 \mathrm{D}$ scanner to get the relevant model, input the data into MATLAB to process and get the relevant movement law, compile the UDF file, then import the data in the file into the model, establish the watershed, divide the grid, set it into FLUENT file, and calculate its hydrodynamic characteristics as shown in Figure 8. 


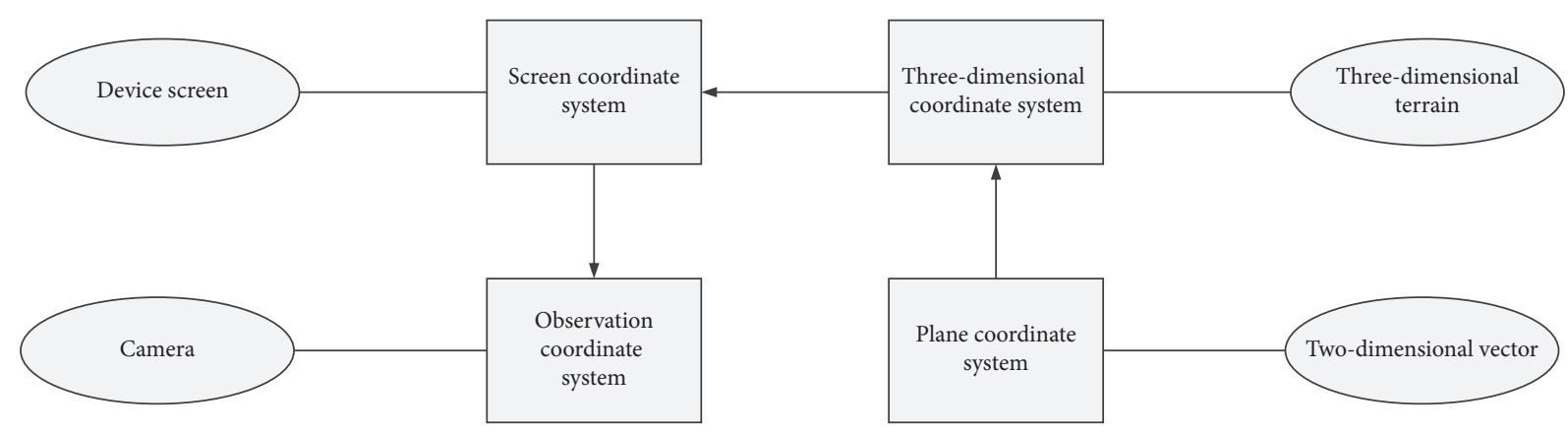

FIgURE 6: Schematic diagram of the coordinate system and its relationship.

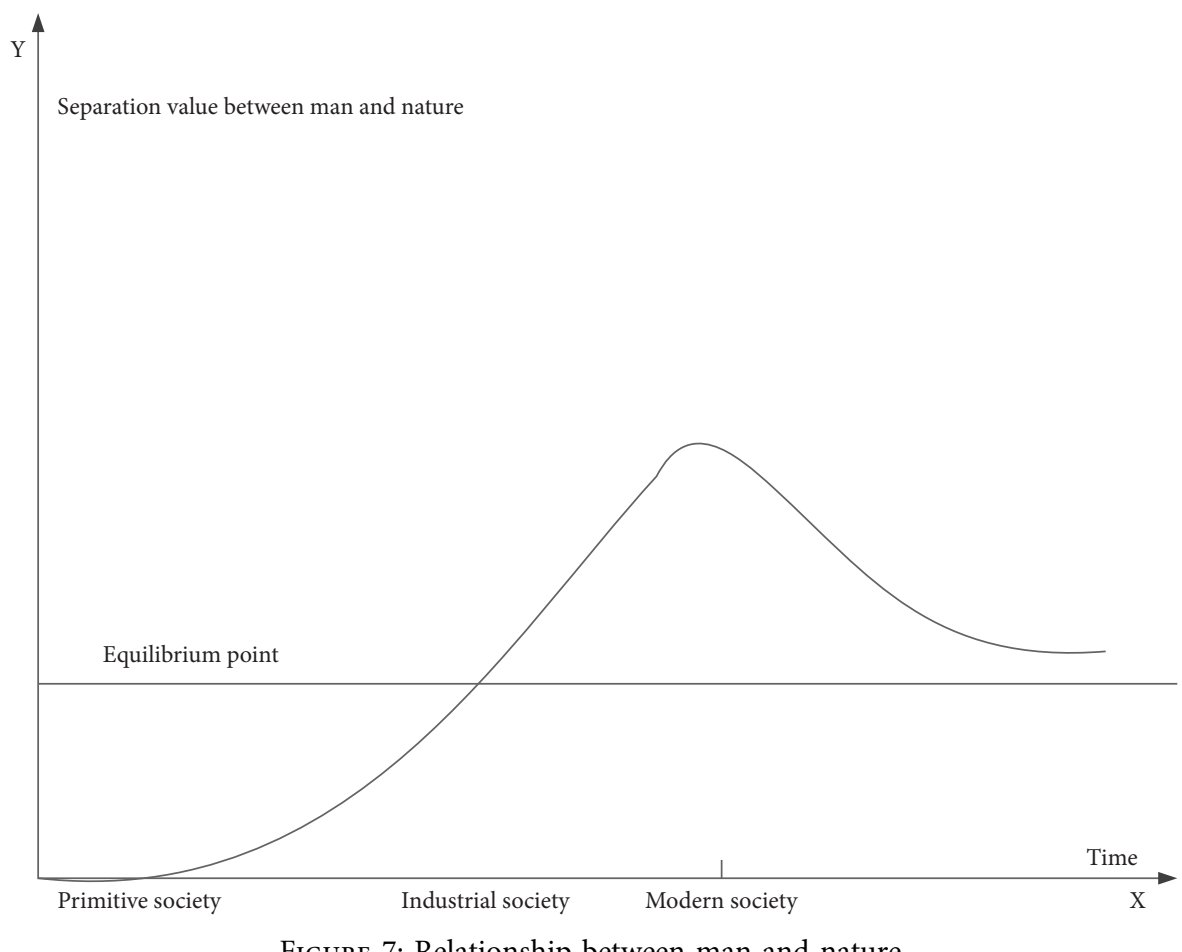

Figure 7: Relationship between man and nature.

\section{Deep Learning and 3D Rendering Model}

3.1. Three-Dimensional Rendering Formula

\subsubsection{Transformation Matrix Calculation}

(1) Camera transformation matrix is as follows:

$$
\begin{gathered}
C M=C^{-1}=(T R)^{-1}=R^{-1} T^{-1}, \\
C M=\left[\begin{array}{cccc}
U_{x} & U_{y} & U_{z} & -U \cdot T \\
V_{x} & V_{y} & V_{z} & -V \cdot T \\
N_{x} & N_{y} & N_{z} & -N \cdot T \\
0 & 0 & 0 & 1
\end{array}\right] .
\end{gathered}
$$

$$
P M=\left[\begin{array}{cccc}
\frac{\cot (1 / 2 \text { fov })}{\text { aspect }} & 0 & 0 & 0 \\
0 & \cot \left(\frac{1}{2} \cdot \text { fov }\right) & 0 & 0 \\
0 & 0 & \frac{Z_{f}}{Z_{f}-Z_{n}} & 1 \\
0 & 0 & \frac{Z_{f} \cdot Z_{n}}{Z_{n}-Z_{f}} & 0
\end{array}\right] .
$$

(2) Perspective projection matrix formula is as follows:

(3) Viewport transformation matrix formula 


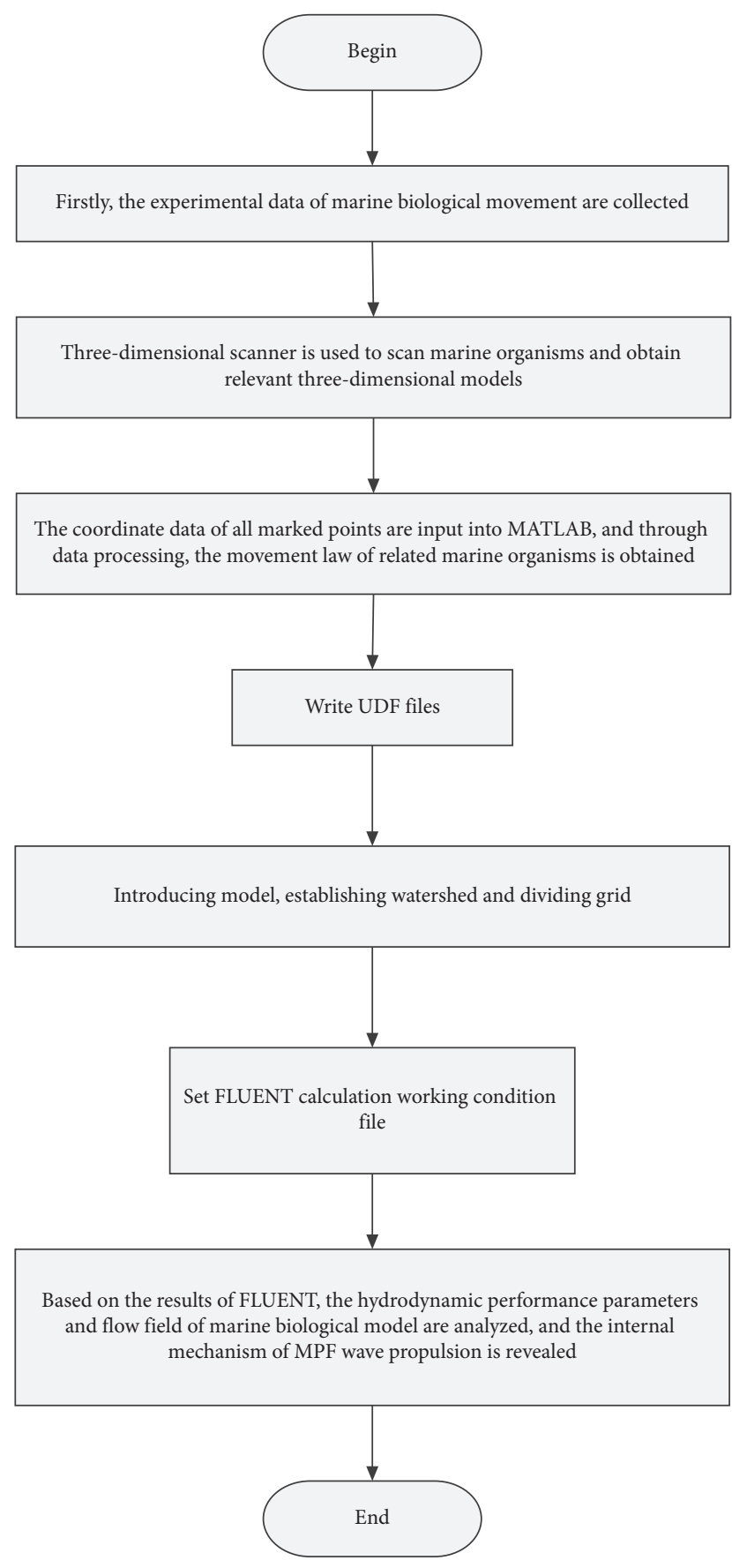

Figure 8: Flow chart of marine bionic packaging container.

\subsection{Deep Learning Formula}

\subsubsection{Score Function}

$S M=\left[\begin{array}{cccc}\frac{\text { Width }}{Z} & 0 & 0 & 0 \\ 0 & -\frac{\text { Height }}{2} & 0 & 0 \\ 0 & 0 & \operatorname{Max} Z-\operatorname{Min} Z & 0 \\ X+\frac{\text { Width }}{2} & Y+\frac{\text { Height }}{2} & \operatorname{Min} Z & 1\end{array}\right]$,

$f(x, W)=W x$.

3.2.2. Calculation of SVM Loss Function. Loss is calculated for categories other than the marked category of the sample; that is, the marked category is not included in the loss, and other categories are calculated and accumulated as the loss of a sample.

$$
L=\frac{1}{N} \sum_{i=1}^{N} \sum_{j \neq y_{i}} \max \left(0, f\left(x_{i}, W\right)_{j}-f\left(x_{i}, W\right) y_{i}+1\right) .
$$

\subsubsection{SVM Loss Function with Regularization Penalty Term} [20]

$L=\frac{1}{N} \sum_{i=1}^{N} \sum_{j \neq y_{i}} \max \left(0, f\left(x_{i}, W\right)_{j}-f\left(x_{i}, W\right) y_{i}+1\right)+\lambda R(W)$.

Among them, $\lambda R(W)$ is the regularization penalty term.

\subsubsection{L2 Regularization}

$$
R(W)=\sum_{K} \sum_{L} W_{K, L}^{2}=\frac{1}{2} W^{2} .
$$

The regularization penalty term SVM loss function is added [21].

$$
L=\text { data_loss }+w_{-} \text {loss. }
$$




\subsubsection{Cross Entropy Loss Function}

$$
L i=-\log \left(\frac{e^{f_{y i}}}{\sum_{j} e^{f_{j}}}\right) .
$$

Among them,

$$
f_{j}(Z)=\frac{e^{Z_{j}}}{\sum_{K} e^{Z_{k}}} .
$$

3.2.6. The Learning Rate of Deep Learning Is the Gradient Descent Step [22]

$$
\frac{\mathrm{d} f(x)}{\mathrm{d} x}=\lim _{h \rightarrow 0} \frac{f(x+h)-f(x)}{h} .
$$

\subsubsection{Single Layer Neural Network[23]}

$$
\begin{aligned}
& f=W_{2} \max \left(0, W_{1} x\right), \\
& f=W_{3} \max \left(0, W_{2} \max \left(0, W_{1} x\right)\right) .
\end{aligned}
$$

\subsubsection{Position Coding}

$$
\begin{aligned}
P E_{(\text {pos }, 2 i)} & =\sin \left(\frac{\text { pos }}{10000^{2 i / d_{\text {model }}}}\right), \\
P E_{(\text {pos }, 2 i+1)} & =\cos \left(\frac{\mathrm{pos}}{10000^{2 i / d_{\text {model }}}}\right), \\
\frac{1}{10000^{2 i / d_{\text {model }}}} & =e^{\log \left\{1 / 10000^{2 i / d_{\text {model }}}\right\}}=e^{-\log \left\{10000^{2 i / d_{\text {model }}}\right\}} \\
& =e^{-2 i / d_{\text {model }} \log \{10000\}}=e^{2 i *\left(\log \{10000\} / d_{\text {model }}\right)} .
\end{aligned}
$$

\subsection{Location Information Embedding Calculation}

$$
\begin{aligned}
v_{2 i} & =\sin \left(\frac{p}{f^{2 i}}\right), \\
v_{2 i+1} & =\cos \left(\frac{p}{f^{2 i}}\right) .
\end{aligned}
$$

$P$ is position information [24]; F is the frequency parameter.

\subsection{Cyclic Neural Network Calculation}

$$
\begin{aligned}
O_{t} & =f\left(V \cdot s_{t}\right), \\
s_{t} & =g\left(U \cdot x_{t}+W \cdot s_{t-1}\right), \\
O_{t} & =f\left(V \cdot g\left(U \cdot X_{t}+W \cdot s_{t-1}\right)\right) .
\end{aligned}
$$

\subsection{Calculation Method of Attention Mechanism}

(1) Point multiplication:

$$
f\left(Q, K_{i}\right)=Q^{T} K_{i}
$$

(2) Weight:

$$
f\left(Q, K_{i}\right)=Q^{T} W K_{i}
$$

(3) Splicing weight:

$$
f\left(Q, K_{i}\right)=W\left[Q^{T} ; K_{i}\right]
$$

(4) Perceptron:

$$
f\left(Q, K_{i}\right)=V^{T} \tanh \left(W Q+U K_{i}\right) .
$$

\section{Research and Test of Deep Learning and 3D Rendering}

\subsection{Research and Test of 3D Rendering}

4.1.1. System Development Test. Through investigation, we know that the test of the $3 \mathrm{D}$ rendering system includes system development environment and system running environment. From these two aspects, we can know that the specific parameters of development environment and running environment are as follows.

It can be seen from the table that the system development environment includes the following four parts, such as system development platform, 3D rendering engine, GPU parallel computing toolset, and terrain data processing toolset. The required parameters are shown in Table 1.

The system operating environment parameters are shown in Table 2.

4.1.2. Factor Influence Test. Influence of Parallel Block Size and Number on $3 D$ Rendering. By studying the influence of the size and number of parallel blocks on the average rendering frame rate, as can be seen from Table 3 , when the number of parallel blocks is 600 and the size is $40 \times 40$, the average rendering frame rate is 13.7 ; when the number of parallel blocks is 1600 and the size is $20 \times 30$, the average rendering frame rate is 16.6; when the number is 2400 and the size is $20 \times 20$, the frame rate is 19.8 and the number is 4800 ; when the size is $10 \times 20$, it is 23.5 , the number is 9600 , and the size is $10 \times 10$. According to the icon and data, it can be seen that with the increase in the number of parallel blocks, the size of parallel blocks decreases and the average rendering frame rate will gradually increase.

Influence of Rendering Window Size and Cooperation on Rendering Frame Rate. The table judges whether the window size affects the rendering frame rate by studying only rendering terrain frame rate, only CPU, and CPU + GPU. From the data in Table 4, it can be seen that when the window size is $600 \times 400$, only the terrain frame rate is 77.3 , and when the window size is $1200 \times 800$, only the terrain frame rate is 68.1 . With the increase in the window size, the terrain frame rate decreases and the 
TABLE 1: System development environment parameters.

\begin{tabular}{lc}
\hline Project & Related data \\
\hline $\begin{array}{l}\text { System development platformThree-dimensional rendering engineGPU Parallel Computing } \\
\text { ToolsetTerrain data processing tool set }\end{array}$ & Visual studio \\
\hline
\end{tabular}

TABLE 2: System running environment parameters.

\begin{tabular}{lc}
\hline Name & Related data \\
\hline Central processing unit & Intel ${ }^{\circledR}$ Xeon ${ }^{\circledR} \mathrm{CPU}$ E5-2609@2.4GHz \\
Graphics processor & Fermi \\
Internal memory & $16 \mathrm{~GB}$ \\
Operating system & Windows7 \\
\hline
\end{tabular}

TABLE 3: Test chart of influence of size and quantity.

\begin{tabular}{lcc}
\hline $\begin{array}{l}\text { Number of parallel } \\
\text { blocks }\end{array}$ & $\begin{array}{c}\text { Parallel block size } \\
(\text { width } \times \text { height })\end{array}$ & $\begin{array}{c}\text { Average rendering } \\
\text { frame rate (FPS) }\end{array}$ \\
\hline 600 & $40 \times 40$ & 13.7 \\
1600 & $20 \times 30$ & 16.6 \\
2400 & $20 \times 20$ & 19.8 \\
4800 & $10 \times 20$ & 23.5 \\
9600 & $10 \times 10$ & 26.9 \\
\hline
\end{tabular}

TABLE 4: Window size image test chart.

\begin{tabular}{lccc}
\hline $\begin{array}{l}\text { Window size } \\
(\text { width } \times \text { length })\end{array}$ & $\begin{array}{c}\text { Render terrain } \\
\text { frame rate }\end{array}$ & $\begin{array}{c}\text { CPU } \\
(\mathrm{fps})\end{array}$ & $\begin{array}{c}\text { CPU + GPU } \\
(\mathrm{fps})\end{array}$ \\
\hline $600 \times 400$ & 77.3 & 1.1 & 29.3 \\
$800 \times 600$ & 75.2 & 1.1 & 26.4 \\
$1024 \times 768$ & 70.1 & 1.0 & 23.3 \\
$1200 \times 800$ & 68.1 & $<1.0$ & 21.5 \\
\hline
\end{tabular}

CPU frame rate also decreases, but the efficiency is low, but when CPU + GPU renders, the efficiency is greatly improved.

It can be seen from Figure 9 that only CPU runtime utility is low, but the rendering frame rate efficiency is greatly improved when CPU and GPU run at the same time.

Influence of Terrain Data Quantity on Rendering Frame Rate. From the data, it can be seen that the number and level have no great influence on the average rendering in Table 5.

Influence of Sampling Point Number on Rendering Performance. The abscissa represents time, and the ordinate represents the rendering frame rate. Figures 10 and 11, respectively, represent two sets of data tests. From the trend of this graph, we can see that the method proposed in this paper is better than the traditional template shadow volume method in rendering and its performance is better. At the same time, the frame rates of MASS, $5 \times 5$ sampling method, and $2 \times 2$ sampling method are all within the acceptable range, and their performance is better. Relatively speaking, the traditional template shadow volume method has lower frame rate and lower performance. $5 \times 5$ sampling and $2 \times 2$ sampling can be selected to ensure good rendering performance as shown in Figures 10 and 11.

4.1.3. Optimization of $3 D$ Rendering Performance of Marine Bionic Packaging Container. Using CARD Model to Optimize $3 D$ Rendering. Three-dimensional modeling refers to the process of building a three-dimensional model on a twodimensional plane. After the creation of the three-dimensional model is completed, it needs to be subjected to material mapping and lighting layout, and the software is used to fit and integrate these with the model to make the three-dimensional model present as real, photo-quality images. This whole process is $3 \mathrm{D}$ modeling and rendering. Table 6 compares simplified and nonsimplified model 3D rendering with a minimum required time of 16.773 and a maximum required time of 18.552 . The average time is 17.773; the minimum required time without simplification is 21.457 , the maximum required time is 26.838 , and the average required time is 24.663 . In contrast, the time required for simplification is significantly lower than that required for nonsimplification. Through the LOD model to improve performance, better three-dimensional rendering of marine life, using the advantages of marine life, and combining with the current environment, the marine bionic packaging container is developed, which makes people develop continuously in the process of getting along with marine life. In order to improve performance, 3D rendering should be simplified as shown in Figure 12.

\subsubsection{Three-Dimensional Rendering Memory Management} Test. By comparing the cached memory management of $3 \mathrm{D}$ rendering with the common memory management, according to the structure, it can be known that the maximum time of cached memory management is $0.87 \mathrm{~s}$ and the minimum time is $1.00 \mathrm{~s}$ when the number of experiments and allocated memory is the same and 1000000 times. Comparing the experimental data, we can see that the time required when using cached memory management is faster than that of ordinary memory management, so using cached memory management can improve the efficiency of $3 \mathrm{D}$ rendering memory management. Therefore, for marine bionics, cache memory management is better than $3 \mathrm{D}$ rendering. Under certain conditions, cache memory management should be preferred as shown in Tables 7 and 8 and Figure 13.

\subsection{Optimization Scheme of Deep Learning Model}

4.2.1. Activation Function. The Sigmoid derivative function moving steeply is not easily derived by comparison and has a nonzero center point as shown in Figures 14 and 15.

Series 1 is sigmoid derivative

Series 2 is a sigmoid function

FIgure 15 shows the tanh function.

Series 1 is tanh function

Series 2 is tanh derivative 


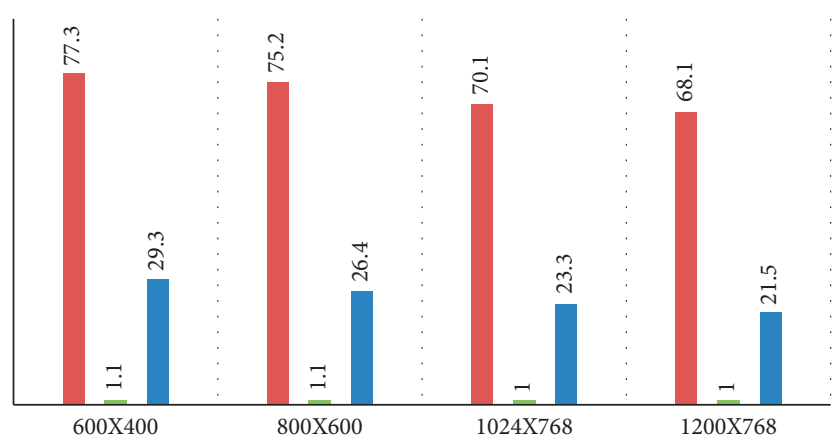

- Render terrain frame rate only

- CPU only

- $\mathrm{CPU}+\mathrm{GPU}$

FIGURE 9: Factors affecting rendering by rendering window size.

TABLE 5: Influence factors of terrain data quantity and level on test.

\begin{tabular}{lcr}
\hline Number of triangular patches of the terrain model & LOD hierarchy of terrain data & Average rendering frame rate (fps) \\
\hline 454 & 1 & 21.5 \\
3023 & 3 & 21.6 \\
1065 & 5 & 22.3 \\
22480 & 9 & 22.1 \\
\hline
\end{tabular}

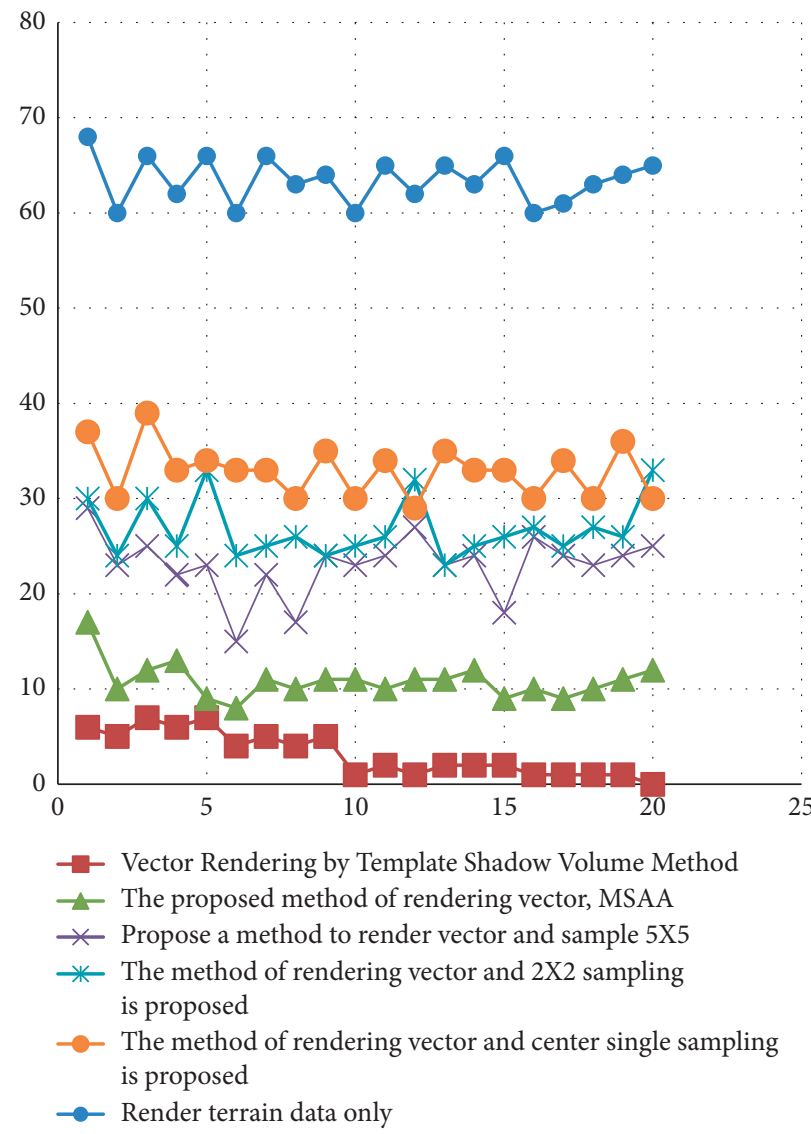

FIGURE 10: Curves of different sampling frame rates by different methods. 


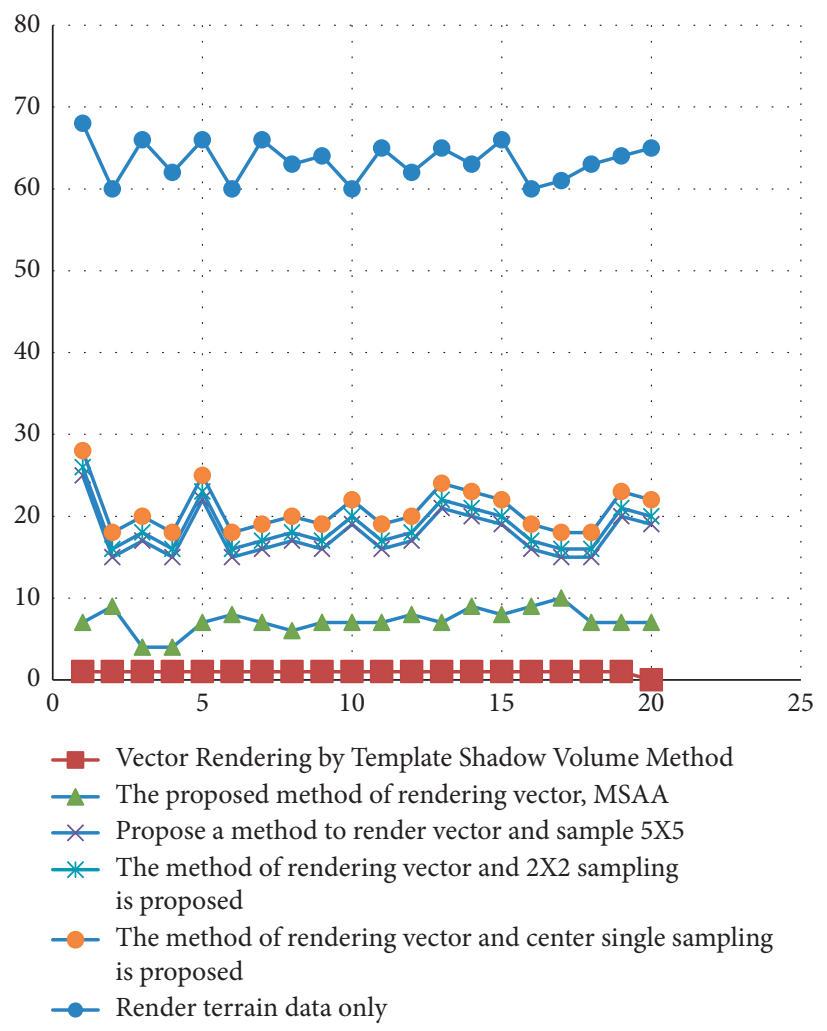

FIGURE 11: Curves of different sampling frame rates by different methods.

TABLE 6: Optimization table of 3D rendering of the LOD model.

\begin{tabular}{lcccccc}
\hline Experimental method & 1 & 2 & 3 & 4 & 5 & Average \\
\hline Not simplified/s & 21.457 & 23.556 & 26.838 & 25.334 & 26.132 & 24.663 \\
Simplify/s & 16.773 & 18.223 & 16.994 & 18.324 & 18.552 & 17.773 \\
\hline
\end{tabular}

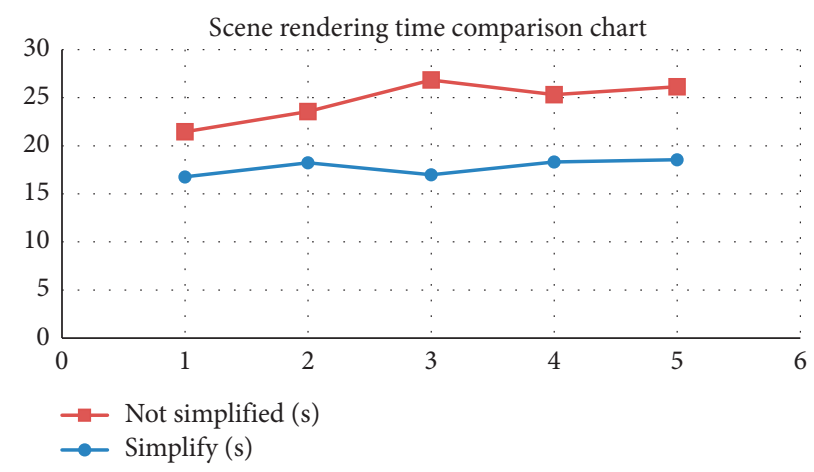

FIGURE 12: LOD model scene rendering time comparison diagram.

TABLE 7: Cached memory management.

\begin{tabular}{lcc}
\hline Number of tests & Number of memory allocations/times & Time spent (s) \\
\hline 1 & 1000000 & 0.87 \\
2 & 1000000 & 1.00 \\
3 & 1000000 & 0.95 \\
4 & 1000000 & 0.88 \\
5 & 1000000 & 0.97 \\
\hline
\end{tabular}


TABLE 8: Normal memory management.

\begin{tabular}{lcc}
\hline Number of tests & Number of memory allocations/times & Time spent (s) \\
\hline 1 & 1000000 & 1.26 \\
2 & 1000000 & 1.45 \\
3 & 1000000 & 1.23 \\
4 & 1000000 & 1.43 \\
5 & 1000000 & 1.38 \\
\hline
\end{tabular}

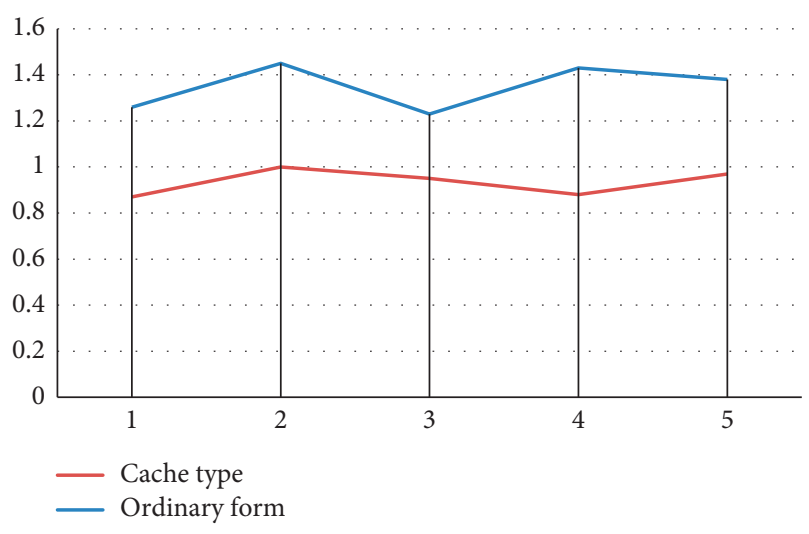

Figure 13: Comparison diagram of 3D rendering memory management.
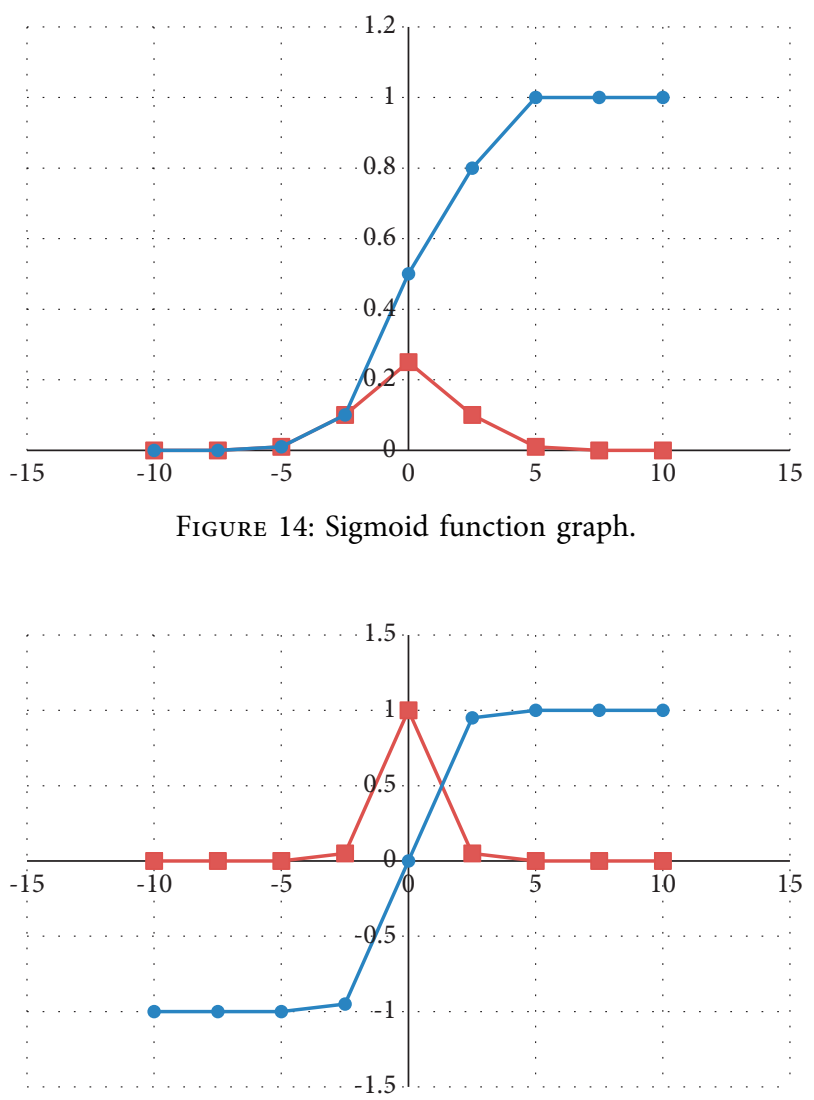

FIgURE 15: Tanh function graph.

A characteristic of that ReLU function is that the value of the function is zero before the coordinate zero. After the zero coordinate point, the trend of one line is more gentle, while the

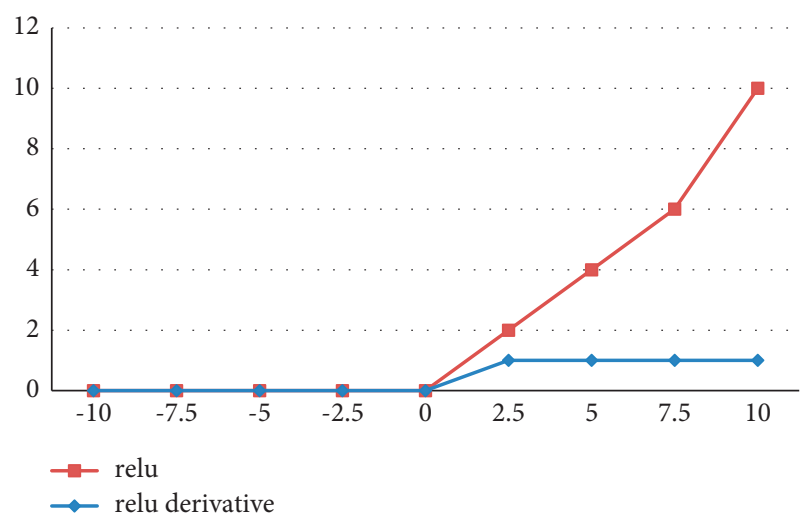

Figure 16: ReLU function graph.

trend of one line is steeper, but it is more accurate than the above two functional models. The optimization model of deep learning neural network is discussed by using the sigmoid derivative function, tanh derivative function, and ReLU function of deep learning neural network. The ReLU function graph is shown in Figure 16.

\section{Conclusion}

Through the combination of $3 \mathrm{D}$ rendering effects, deep neural networks, and visualization methods, the $3 \mathrm{D}$ rendering of deep learning and visualization of marine bionic packaging containers is analyzed. Through testing the three-dimensional rendering, the influencing factors include the size and number of parallel blocks, the size of the rendering window, the amount of terrain data, and the number of sampling points; according to the relevant influencing factors, the LOD model is used to optimize the three-dimensional rendering. Through the optimization of the deep learning model, the three activation functions are studied, and the advantages and disadvantages are analyzed. Through the research in this article, the relevant advantages of marine organisms can be used to create marine bionic packaging containers suitable for human use, which will make our lives more convenient.

\section{Data Availability}

The experimental data used to support the findings of this study are available from the corresponding author upon request.

\section{Conflicts of Interest}

The authors declare that they have no conflicts of interest regarding this work. 


\section{Acknowledgments}

This work was supported by a grant from Brain Korea 21 Program for Leading Universities and Students (BK21 FOUR) MADEC Marine Designeering Education Research Group.

\section{References}

[1] T. D. Le and H. V. Pham, Intelligent data analysis: deep learning and visualization, Springer, Berlin, Germany, pp. 85-114, 2020.

[2] G. Sun and Q. Qian, "Deep learning and visualization for identifying malware families," IEEE Transactions on Dependable and Secure Computing, vol. 8, no. 1, pp. 283-295, 2018.

[3] R. T. Schirrmeiste, J. T. Springenberg, L. Fiedere et al., "Deep learning with convolutional neural networks for EEG decoding and visualization," Human Brain Mapping, vol. 3, no. 3, pp. 21-23, 2017.

[4] M. A. Fredericksen, Y. Zhang, M. L. Hazen et al., "Threedimensional visualization and a deep-learning model reveal complex fungal parasite networks in behaviorally manipulated ants," Proceedings of the National Academy of Sciences, vol. 114, no. 47, pp. 12590-12595, 2017.

[5] H. L. Liu, T. Taniguchi, Y. Tanaka, K. Takenaka, and T. Bando, "Visualization of driving behavior based on hidden feature extraction by using deep learning," IEEE Transactions on Intelligent Transportation Systems, vol. 18, no. 9, pp. 2477-2489, 2017.

[6] S. Ni, Q. Qian, and R. Zhang, "Malware identification using visualization images and deep learning," Computers \& Security, vol. 77, no. AUG, pp. 871-885, 2018.

[7] V Gulshan, L Peng, M Coram et al., "Development and validation of a deep learning algorithm for detection of diabetic retinopathy in retinal fundus photographs," Jama, vol. 65 , no. 23 , pp. $134-145,2016$.

[8] L. Banko, P. M. Maffettone, D. Naujoks, D. Olds, and A. Ludwig, "Deep learning for visualization and novelty detection in large X-ray diffraction datasets," Npj Computational Materials, vol. 7, no. 5, pp. 47-65, 2021.

[9] Z. Zhuang, G. Liu, W. Ding et al., "Cardiac VFM visualization and analysis based on YOLO deep learning model and modified 2D continuity equation," Computerized Medical Imaging and Graphics, vol. 82, Article ID 101732, 2020.

[10] T. Zebin, S. Rezvy, and P. Wei, "COVID-19 detection and disease progression visualization: deep learning on chest $\mathrm{X}$-rays for classification and coarse localization," Applied Intelligence, vol. 51, no. 6, 2021.

[11] W. Zhu, Z. T. Webb, K. Mao, and J. Romagnoli, “A deep learning approach for process data visualization using t-distributed stochastic neighbor embedding," Industrial \& Engineering Chemistry Research, vol. 58, no. 22, 2019.

[12] U. Tiede, K. H. Hoehne, M. Bomans, A. Pommert, M. Riemer, and G. Wiebecke, "Investigation of medical 3D-rendering algorithms," IEEE Computer Graphics and Applications, vol. 10, no. 2, pp. 41-53, 1990.

[13] R. H. Choplin, K. A. Buckwalter, J. Rydberg, and J. M. Farber, "CT with 3D rendering of the tendons of the foot and ankle: technique, normal anatomy, and disease," RadioGraphics, vol. 24, no. 2, pp. 343-356, 2004.

[14] H. Henkes, G. Huber, J. Hierholzer, M. Cordes, C. Kujat, and U. Piepgras, "Radionuclide cisternography, using SPECT and 3D rendering," Computer Assisted Radiology/Computergestützte Radiologie, vol. 32, no. 1, pp. 43-56, 1991.
[15] P. Zanuttigh and G. M. Cortelazzo, "Compression of depth information for 3D rendering," in Proceedings of the $3 D T V$ conference: the true vision - capture, transmission and display of 3D video, vol. 21, no. 3, pp. 65-78, IEEE, Copenhagen, 2009.

[16] G. E. Dahl, D. Yu, L. Deng, and A. Acero, "Context-dependent pre-trained deep neural networks for large-vocabulary speech recognition," IEEE Transactions on Audio Speech and Language Processing, vol. 20, no. 1, pp. 30-42, 2011.

[17] G. Dennis, B. T. Sherman, D. A. Hosack et al., "DAVID: database for annotation, visualization, and integrated discovery," Genome Biology, vol. 4, no. 9, pp. 108-119, 2003.

[18] P. Diazgutierrez, A. Bhushan, R. Pajarola, and M Gopi, "Constrained strip generation and management for efficient interactive 3D rendering," in Proceedings of the Computer Graphics International 2005, vol. 32, no. 5, pp. 66-83, IEEE, Washington, DC, USA, June 2005.

[19] V. Verma and W. Ekta, "3D rendering - techniques and challenges," International Journal of Engineering \& Technology, vol. 2, no. 2, 2010.

[20] J. P. Shanjiang and J.C.. Online, "LS-SVM for function estimation and classification," Journal of University of ence \& Technology Bjing, vol. 10, no. 5, pp. 73-77, 2003.

[21] J. Schmidhuber, "Deep learning in neural networks: an overview," Neural Networks, vol. 61, pp. 85-117, 2015.

[22] S. L. Lin and Z. Liu, "Parameter selection in SVM with RBF kernel function," Journal of Zhejiang University of Technology, vol. 65, no. 3, pp. 76-98, 2007.

[23] O. Fontenla-Romero, B. Guijarro-Berdiñas, B. Pérez-Sánchez, and A. Alonso-Betanzos, "A new convex objective function for the supervised learning of single-layer neural networks," Pattern Recognition, vol. 43, no. 5, pp. 1984-1992, 2010.

[24] X. Xian Wang, X. Xianfu Lei, P. Pingzhi Fan, R. Q. Hu, and fnm Shi-Jinn Horng, "Cost analysis of movement-based location management in PCS networks: an embedded Markov chain approach," IEEE Transactions on Vehicular Technology, vol. 63, no. 4, pp. 1886-1902, 2014. 